Kragujevac Journal of Mathematics

Volume 45(5) (2021), Pages 781-786.

\title{
APPLICATION OF THE HOPF MAXIMUM PRINCIPLE TO THE THEORY OF GEODESIC MAPPINGS
}

\author{
SERGEY STEPANOV ${ }^{1,2}$ AND JOSEF MIKEŠ ${ }^{3}$
}

\begin{abstract}
In the present paper we consider some applications the Hopf maximum principle and its generalization to the classical theory of geodesic mappings. As a result, a series of classical theorems on geodesic mappings become consequences of our statements which we shall prove in the present paper.
\end{abstract}

\section{INTRODUCTION}

The Hopf maximum principle is a maximum principle in the theory of second order elliptic differential equations and has been described as the "classic and bedrock result" of that theory. E. Hopf proved in 1927 that if a function satisfies a second order partial differential inequality of a certain kind in a connected domain of $\mathbb{R}^{n}$ and attains a maximum in the domain then the function is constant. The simple idea behind Hopf's proof, the comparison technique he introduced for this purpose, has led to an enormous range of important applications and generalizations (see $[2,3,14]$ ). In the present paper we consider some applications the Hopf maximum principle and its generalization to the classical theory of geodesic mappings or in other words projective mappings (see, for example, [5, p. 131-142], [9-11]). As a result, a series of classical theorems on geodesic mappings become consequences of our statements which we shall prove in the present paper.

Key words and phrases. Riemannian manifold, Einstein manifold, geodesic mapping, second order elliptic differential operator on symmetric tensors, Hopf maximum principle, vanishing theorems.

2010 Mathematics Subject Classification. Primary: 53C20, 53B20. Secondary: 53C21, 53C24.

DOI 10.46793/KgJMat2105.781S

Received: June 19, 2018.

Accepted: May 20, 2019. 


\section{Geodesically Equivalent Riemannian Metrics on Complete and Compact Riemannian Manifolds}

Two Riemannian metrics $g$ and $\bar{g}$ on a connected domain $U \subset M$ of a same smooth manifold $M$ are said to be pointwise projectively equivalent or in other words pointwise geodesically equivalent, if every geodesic of $g$ in $U$ is a reparametrized geodesic of $\bar{g}$. In addition, we say that $g$ and $\bar{g}$ are pointwise affine equivalent in a connected domain $U \subset M$, if their Levi-Civita connections $\nabla$ and $\bar{\nabla}$ of $g$ and $\bar{g}$ respectively, coincide.

The volume element of $g$ is the volume form $\operatorname{Vol}(g)$, which is defined whether or not $M$ is oriented. In local coordinates, $\operatorname{Vol}(g)=\sqrt{\operatorname{det} g}|d x|$. In turn, for $\bar{g}$ we have $\operatorname{Vol}(\bar{g})=\sqrt{\operatorname{det} \bar{g}}|d x|$. As well known (see [5, p. 133]), two metrics $g$ and $\bar{g}$ are geodesically equivalent in a connected domain $U \subset M$ if and only if for the function

$$
\varphi=\frac{1}{n+1} \log \left(\frac{\operatorname{Vol}(\bar{g})}{\operatorname{Vol}(g)}\right)
$$

we have

$$
\left(\nabla_{Z} \bar{g}\right)(X, Y)=2 \bar{g}(X, Y) d \varphi(Z)+\bar{g}(X, Z) d \varphi(Y)+\bar{g}(Y, Z) d \varphi(X)
$$

at every point $x$ of $U \subset M$ and for any vectors $X, Y, Z \in T_{x} M$. As a consequence of these equations, we obtain the following equalities (see [5, p. 135])

$$
\overline{\operatorname{Ric}}=\operatorname{Ric}+(n-1)(\nabla d \varphi-d \varphi \otimes d \varphi),
$$

where Ric and $\overline{\text { Ric }}$ are the Ricci tensors of $g$ and $\bar{g}$, respectively. Now, if we set $\Delta \varphi=$ trace $_{g} \nabla d \varphi$, then from (2.3) have

$$
\Delta \varphi=\frac{1}{n-1}\left(s^{*}-s\right)+g(d \varphi, d \varphi),
$$

for $\|\varphi\|^{2}=g(d \varphi, d \varphi)$, the scalar curvature $s=$ trace $_{g}$ Ric of $g$ and $s^{*}=$ trace $_{g} \overline{\mathrm{Ric}}$.

Now, we prove the following theorem.

Theorem 2.1. Let $g$ and $\bar{g}$ be two pointwise geodesically equivalent Riemannian metrics on a connected domain $U \subset M$ of an $n$-dimensional $(n \geq 2)$ smooth manifold $M$ such that $s^{*} \geq s$ at every point of $U$, where $s$ is the scalar curvature of $g$ and $s^{*}=$ trace $_{g} \overline{\operatorname{Ric}}$ for the Ricci tensor $\overline{\operatorname{Ric}}$ of $\bar{g}$. The assumption that the function $\varphi=(n+1)^{-1} \log (\operatorname{Vol}(\bar{g}) / \operatorname{Vol}(g))$ attains a local maximum value at some point $x \in U$ implies that $g$ and $\bar{g}$ are geodesically equivalent on $U$ if and only if they are pointwise affinely equivalent metrics. Furthermore, if there is at least one point of $U$ at which $s^{*}>s$, then $\bar{g}=g$.

Proof. We suppose now that $g$ and $\bar{g}$ be two geodesically equivalent Riemannian metrics on a connected domain $U \subset M$ of an $n$-dimensional smooth manifold $M$ such that $s^{*} \geq s$ where $s$ is the scalar curvature of $g$ and $s^{*}=$ trace $_{g} \overline{\text { Ric }}$ for the Ricci tensor $\overline{\operatorname{Ric}}$ of $\bar{g}$. As a result, the function $\varphi=(n+1)^{-1} \log (\operatorname{Vol}(\bar{g}) / \operatorname{Vol}(g))$ satisfies the inequality $\Delta \varphi \geq 0$ at each point of $U$, by (2.4). Therefore, $\varphi$ is a subharmonic function (see $[3,14])$. In this case, assumption that the function $\varphi$ attains a local 
maximum value at some point then implies $\varphi$ is a constant $C$ in $U$, by the Hopf's maximum principle (see [3, Theorem 1]). Then from $(2.2)$ we obtain that $\nabla \bar{g}=0$ on $U$ and hence $g$ and $\bar{g}$ are affine equivalent on $U$. If $C>0$, then $\operatorname{grad} \varphi$ is nowhere zero. Now, at a point where $s^{*}>s$, the left side of (2.4) is zero while the right side is positive. This contradiction shows that $C=0$ and hence $\bar{g}=g$. Thus we have proved our Theorem 2.1.

In particular, if $\overline{\operatorname{Ric}} \geq 0$ and $s \leq 0$ at an arbitrary point of $U$ then $s^{*} \geq s$. In this case, $\Delta \varphi \geq 0$ at each point of $U$, by (2.4). Therefore, the following corollary is true.

Corollary 2.1. Let $g$ and $\bar{g}$ be two Riemannian metrics on a connected domain $U \subset M$ of an $n$-dimensional $(n \geq 2)$ compact smooth manifold $M$ such that $s \leq 0$ for the scalar curvature $s$ of $g$ and $\overline{\mathrm{Ric}} \geq 0$ for the Ricci tensor $\overline{\mathrm{Ric}}$ of $\bar{g}$. Then the assumption that the function $\varphi=(n+1)^{-1} \log (\operatorname{Vol}(\bar{g}) / \operatorname{Vol}(g))$ attains a local maximum value at some point $x \in U$ implies that $g$ and $\bar{g}$ are pointwise geodesically equivalent if and only if they are pointwise affinely equivalent metrics. Furthermore, if there is at least one point $x \in U$ at which the Ricci tensor $\overline{\operatorname{Ric}}$ is positive in all directions or the scalar curvature $s$ is negative, then $\bar{g}=g$.

Let $U=M$ and $M$ be a compact manifold. Then there exists a point $x \in M$ at which the function $\varphi=(n+1)^{-1} \log (\operatorname{Vol}(\bar{g}) / \operatorname{Vol}(g))$ attains the maximum. As a result we can formulate the following statements that are corollaries of our Theorem 2.1 (see also Theorem 3 and Corollary 4 from [7] and with Theorem 1.3 from [4]).

Corollary 2.2. Let $g$ and $\bar{g}$ be two Riemannian metrics on an $n$-dimensional $(n \geq 2)$ compact smooth manifold $M$ such that $s^{*} \geq s$ where $s$ is the scalar curvature of $g$ and $s^{*}=$ trace $_{g} \overline{\mathrm{Ric}}$ for the Ricci tensor $\overline{\mathrm{Ric}}$ of $\bar{g}$. Then $g$ and $\bar{g}$ are pointwise geodesically equivalent if and only if they are pointwise affinely equivalent metrics. Furthermore, if there is at last point of $M$ at which $s^{*}>s$, then $\bar{g}=g$.

Corollary 2.3. Let $g$ and $\bar{g}$ be two geodesically equivalent Riemannian metrics on an $n$-dimensional compact smooth manifold $M$ such that $s \leq 0$ and $\overline{\text { Ric }} \geq 0$ where $s$ is the scalar curvature of $g$ and $\overline{\mathrm{Ric}}$ is the Ricci tensor of $\bar{g}$. Then $g$ and $\bar{g}$ are pointwise geodesically equivalent if and only if they are pointwise affinely equivalent metrics. Furthermore, if there is at least one point of $M$ at which the Ricci curvature $\overline{\text { Ric }}$ is positive or the scalar curvature $s$ is negative, then $\bar{g}=g$.

Let $g$ and $\bar{g}$ be two geodesically equivalent Riemannian metrics on a connected domain $U \subset M$ of an $n$-dimensional $(n \geq 2)$ smooth manifold $M$. We suppose that $\operatorname{grad} \varphi=\left(\varphi_{i}\right)$ and $\bar{g}^{-1}=\left(\bar{g}^{j k}\right)$ with respect to a local coordinate system $x^{1}, \ldots, x^{n}$ on $U$ and denote by $\xi$ the vector field with the local components $\xi^{j}=\varphi_{k} \bar{g}^{j k}$ for $i, j, k=1, \ldots, n$. If the metric $g$ is an Einstein metric then by direct calculations we obtain the formula (see also [12])

$$
\Delta \varphi=\frac{2(n+3)}{n(n-1)} s \cdot \psi+2 g(\nabla \xi, \nabla \xi),
$$


for $\psi=e^{4 \varphi} g(\xi, \xi)$. This formula is an analogue of our formula (2.4). Therefore, we can prove an analogue of our Theorem 2.1.

Theorem 2.2. Let $g$ be an Einstein metric with the nonnegative scalar curvature $s$ on a connected domain $U \subset M$ of an $n$-dimensional $(n \geq 3)$ smooth manifold $M$. If there exists another Riemannian metric $\bar{g}$ on $U$ that pointwise geodesically equivalent to $g$ and the function $\psi=e^{4 \varphi} g(\xi, \xi)$ for the vector field $\xi$ corresponding to $\operatorname{grad} \varphi$ under the duality defined by the metric $\bar{g}$ attains a local maximum value at some point $x \in U$, then the scalar curvature $s$ is necessarily equal to zero and $\bar{g}$ is pointwise affine equivalent to $g$ or $\bar{g}=g$ for the case $s>0$.

Let $U=M$ and $M$ be a compact smooth manifold. Then there exists a point $x \in M$ at which the function $\psi$ attains the maximum. As a result we can formulate the following theorem that is a corollary of our Theorem 2.2 (see also [12]).

Corollary 2.4. Let $M$ be an $n$-dimensional $(n \geq 3)$ compact smooth manifold $M$ and $g$ be an Einstein metric with nonnegative scalar curvature $s$ on $M$. If there exists another Riemannian metric $\bar{g}$ on $M$ that pointwise geodesically equivalent to $g$, then the scalar curvature $s$ is necessarily equal to zero and $\bar{g}$ is pointwise affine equivalent to $g$ or $\bar{g}=g$ for the case $s>0$.

\section{Geodesically Equivalent Riemannian Metrics on Complete Noncompact Riemannian Manifolds}

$\mathrm{Li}$ and Schoen have proved in [8] that there is no a non-constant, non-negative $L^{p}$-integrable $(0<p<\infty)$ subharmonic function $\psi$ on any complete Riemannian manifold $(M, g)$ with non-negative Ricci tensor. In other word, if we suppose that Ric $\geq 0$ and $\int_{M}\|\psi\|^{p} d \operatorname{Vol}_{g}<\infty$ for a complete Riemannian manifold $(M, g)$, then $\psi=C$ for some constant $C$. In this case, we have $C^{p} \int_{M} d \mathrm{Vol}_{g}<\infty$. If $C>0$, $\psi$ is nowhere zero and the volume of $(M, g)$ is finite. Side by side, we know from [14] that every complete non-compact Riemannian manifold $(M, g)$ with non-negative Ricci tensor has infinite volume. This contradiction shows $C=0$ and hence $\psi \equiv 0$. Therefore, we can formulate the following lemma.

Lemma 3.1. Let $(M, g)$ be a complete non-compact Riemannian manifold with nonnegative Ricci tensor, then there is no nonzero non-negative $L^{p}(M, g)$-integrable $(0<$ $p<\infty)$ subharmonic function.

On the other hand, if the scalar curvature $s$ of an Einstein metric $g$ is nonnegative then $\operatorname{Ric}=\frac{s}{n} g \geq 0$ and from (2.5) we obtain $\Delta \psi \geq 0$ and hence $\psi$ is a non-negative subharmonic function.

Using the Lemma we can formulate the following statement.

Corollary 3.1. Let $(M, g)$ be a complete non-compact Einstein manifold with nonnegative scalar curvature, and $\bar{g}$ be another Riemannian metric on $M$ that pointwise geodesically equivalent to $g$. If the function $\psi=e^{4 \varphi} g(\xi, \xi)$ for the vector field $\xi$ 
corresponding to $\operatorname{grad} \varphi$ under the duality defined by the metric $\bar{g}$ is $L^{p}(M, g)$-integrable $(0<p<\infty)$ function then the scalar curvature $s$ is necessarily equal to zero and $\bar{g}$ is pointwise affine equivalent to $\mathrm{g}$.

Remark 3.1. Other results on pointwise geodesically equivalent Riemannian metrics on compact and non-compact Riemannian manifolds can be found among others in papers from the following list $[1,6,9,12,13]$.

Acknowledgements. Research of the second author was supported by project IGA PrF 2019015 Palacky University Olomouc.

\section{REFERENCES}

[1] I. A. Aleksandrova, J. Mikeš, S. E. Stepanov and I. I. Tsyganok, Liouville type theorems in the theory of mappings of complete Riemannian manifolds, J. Math. Sci. (N.Y.) 221 (2017), 737-744.

[2] S. Bochner and K. Yano, Curvature and Betti Numbers, Princeton University Press, Princeton, 1953.

[3] E. Calabi, An extension of E. Hopf's maximum principle with an application to Riemannian geometry, Duke Math. J. 25 (1958), 45-56.

[4] X. Chen and Z. Shen, A comparison theorem on the Ricci curvature in projective geometry, Ann. Global Anal. Geom. 23 (2003), 141-155.

[5] L. P. Eisenhart, Riemannian Geometry, Princeton University Press, Princeton, New Jersey, 1949.

[6] I. Hinterleitner, Geodesic mappings on compact Riemannian manifolds with conditions on sectional curvature, Publ. Inst. Math. (Beograd) (N.S.) 94(108) (2013), 125-130.

[7] S. Kim, Volume and projective equivalence between Riemannian manifolds, Ann. Global Anal. Geom. 27 (2005), 47-52.

[8] P. Li and R. Schoen, $L^{p}$ and mean value properties of subharmonic functions on Riemannian manifolds, Acta Math. 153 (1984), 279-301.

[9] J. Mikeš, E. Stepanova, A. Vanžurová and et al., Differential Geometry of Special Mappings, Palacký University Olomouc, Faculty of Science, Olomouc, 2015.

[10] J. Mikeš, A. Vanžurová and I. Hinterleitner, Geodesic Mappings and some Generalizations, Palacký University Olomouc, Faculty of Science, Olomouc, 2009.

[11] N. S. Sinyukov, Geodesic Mappings of Riemannian Spaces, Nauka, Moscow, 1979.

[12] E. N. Sinyukova, On the geodesic mappings of some special Riemannian spaces, Math. Notes 30 (1981), 946-949.

[13] S. E. Stepanov, I. I. Tsyganok and J. Mikeš, A Liouville type theorem on the projective mapping of a complete Riemannian manifold, Differentsial'naya Geom. Mnogoobraz. Figur (2017), 110 115.

[14] S. T. Yau, Some function-theoretic properties of complete Riemannian manifold and their applications to geometry, Indiana Univ. Math. J. 25 (1976), 659-670.

\footnotetext{
${ }^{1}$ Department of Mathematics,

All Russian Institute for Scientific and Technical Information

OF THE Russian ACADEMY OF SCIENCES,

Usievicha STREet 20, 125190 Moscow, Russia
} 
${ }^{2}$ Department of Mathematics,

Finance University under the Government of the Russian Federation, Leningradsky Prospect 49-55, 125468 Moscow, Russia

Email address: s.e.stepanov@mail.ru

${ }^{3}$ Department of Algebra and Geometry,

PALACKY UNIVERSITY,

17. listopadu 12, 77146 Olomouc, Czech Republic

Email address: josef.mikes@upol.cz 